\title{
Tangence
}

\section{Le récit criminel}

\section{Simon Harel}

Numéro 37, septembre 1992

Autopsie du fait divers

URI : https://id.erudit.org/iderudit/025728ar

DOI : https://doi.org/10.7202/025728ar

Aller au sommaire du numéro

Éditeur(s)

Tangence

ISSN

1189-4563 (imprimé)

1710-0305 (numérique)

Découvrir la revue

Citer cet article

Harel, S. (1992). Le récit criminel. Tangence, (37), 87-96.

https://doi.org/10.7202/025728ar d'utilisation que vous pouvez consulter en ligne.

https://apropos.erudit.org/fr/usagers/politique-dutilisation/ 


\section{Le récit criminel}

\section{Simon Harel *}

Le fait divers en cause ici nous amène à percevoir l'irrémédiable. C'est du moins le cas du discours qui impose la mise à mort comme un acte dont l'irruption dans la vie sociale est monstrueuse. J'avoue d'emblée mon malaise. Que dire de ce texte dont la « rumeur * folle, à propos du meurtre perpétré, est le seul référent dont je puisse cerner l'existence. Il y a bien eu un meurtre réel dont je connais l'existence par le biais d'un discours rapporté. Mais ce meurtre est autre chose qu'un objet discursif qu'il s'agit, purement et simplement, d'analyser. Il représente l'horreur même et l'insanité qui l'accompagne. À titre de lecteur, quelle est la position éthique qui accompagne l'analyse que je proposerai ici? Dois-je me contenter de soupeser l'argumentation de même que les diverses configurations textuelles qui donnent à ce discours sa pragmatique? Mais ce faisant, je laisse de côté la souffrance et la violence qui sont incarnées dans ce fait divers et je cours le risque de banaliser l'irrémédiable pour mieux en offrir une analyse conforme, susceptible d'expliquer la genèse du trouble que ce texte évoque en moi. Par contre, peut-on demeurer silencieux devant un tel écrit? N'est-il pas au contraire urgent de décortiquer tout discours qui valorise l'immédiateté persuasive, la transmission inaltérée de la "rumeur* publique? Le statut du lecteur critique est donc ambigu. D'une part, il craint de surestimer la matérialité argumentative qui * construit " littéralement l'élaboration du fait divers. Car la perspective d'analyse invoquée peut rejeter la trame personnelle - même si elle est déjà socialisée - qui est à l'origine de ce drame. Que savons-nous en effet de ce meurtre, de sa justification, des événements qui ont provoqué l'actualisation de la pulsion homicide? En somme, que peut révéler d'intime le fait divers qui condamne le lecteur au voyeurisme et à la jouissance différée d'être le spectateur de la mort d'autrui? D'autre part, malgré toute sa probité, le lecteur ne peut éviter ce singulier passage à l'acte que représente aussi, à sa manière, le discours critique. Face à l'inertie doxologique, il faut scruter, jauger le discours social.

- Université du Québec à Montréal. 
88

Pourquoi cet adolescent a-t-il tué ses parents, un de ses frères et une de ses sours? Quelle folle dérive a bien pu le laisser commettre l'irrémédiable? C'est la première question qui me vient à l'esprit, absurde, en tout cas incongrue. Cette famille dévastée par la violence meurtrière, elle est l'exemple même de l'anéantissement du socius. L'adolescent tue ses géniteurs, son frère et sa soeur. Il détruit ceux qui l'ont précédé en lui donnant naissance. Il met à mort la fratrie qui l'a accompagné en souhaitant faire table rase par l'appel à la pulsion homicide. Tuer, condamner, disparaître, telle est la culture banalisée de la pulsion de mort que nous sommes amenés à constater quotidiennement.

J'imagine que ce fait divers est digne de mention et mérite d'être analysé parce qu'il trouve sa source dans une interrogation sur l'inscription de la mort dans le discours social. Quel lecteur troublé peut bien vouloir se prêter à pareille démarche analytique? J'ajouterais: quelle complaisance - qui va jusqu'au désistement critique - peut pousser à rendre compte de la discursivité factice de ce fait divers? Peut-on analyser objectivement les figures de la mort d'autrui? Ma première attitude - celle du lecteur qui constate le caractère platement référentiel du fait divers - en est une de colère. C'est que le discours social recourt aisément aux images de débordement passionnel, aux figures de l'excès qui désignent, selon moi, autant de passages à l'acte socialisés que nous subissons. En somme, le fait divers, tel qu'on l'observe ici, c'est l'acceptation de la folie paranoïde. Un tel discours affirme: "Soyez sur vos gardes. En chacun de vous sommeille un monstre. Et vos enfants que vous croyez dociles... Ne vous méprenez pas: ils sont menaçants. Un jour viendra où ils vous tueront*. Mais ici la difformité ne prend plus l'aspect de la déficience mentale, de la laideur corporelle, de l'anomalie. La monstruosité - qui signifie toujours l'envers de la régularité - a le visage d'un adolescent dont on ne peut dire ce qui le distingue des autres ${ }^{1}$. Dans ce fait divers, les marqueurs de la différence sont risibles tant il construisent une identité de surface:

Le garçon, qui s'était en partie rasé la tête et qui avait teint le reste de ses cheveux en noir avant que les cadavres ne soient découverts, était en cours d'interrogatoire à la poste centrale de Rochester, une ville de 60000 habitants.

1 Sur cette modification des paramètres sociaux et culturels de la monstruosité, on consultera l'ouvrage de Gilbert Lascault, Le monstre dans l'art occidental. Un problème esthétique, Paris, Klincksieck, 1973. 
Voilà donc le meurtrier: un enfant criminel dont la différence est emblématisée par un rituel dérisoire qui accompagne la mise à mort des siens. Un rituel dont est absente toute impulsion tragique. Quoiqu'une certaine dérision apocalyptique accompagne la description de ce fait divers. On est bien loin de l'affirmation d'un Jean Genet pour lequel ce qui conduit les enfants au crime:

[...] c'est le sentiment romanesque, c'est-à-dire la projection de soi dans la plus magnifique, la plus audacieuse, enfin la plus périlleuse des vies. Je traduis pour eux, car ils ont le droit d'utiliser un langage qui les aide à s'aventurer... Où croyez-vous? Je ne sais pas. Ils ne le savent pas non plus, même si leur rêverie se veut précisée, mais c'est hors de chez vous. ${ }^{2}$

S'il y a une dimension apocalyptique dans la présentation de ce fait divers, celle-ci est anonyme, banale. Pourtant, moi qui scrute ce texte aujourd'hui, j'ai appris, comme vous sans doute, et dans les mêmes journaux, qu'un adolescent a tué en mai sa famille à Montréal. S'agit-il d'un fait divers? La répétition de la pulsion homicide est-elle plus supportable parce qu'elle est empreinte de banalité? Il faut sans doute, c'est la position du lecteur inquiet qui déteste la suffisance du fait divers, fonder du sens, octroyer une lisibilité à ce qui demeure l'expression d'un brouillage médiatique insupportable: la mise à nu de l'agression au nom du pouvoir de vie ou de mort qu'un sujet détient sur autrui.

Pour contrecarrer la proximité de la mort qui hante ce fait divers, il faut aussi - du moins, c'est ma stratégie - chercher parmi des références personnelles: un souvenir, un rêve, une lecture, une évocation, une réflexion qui permettent de taire la numeur anonyme qui transmet la réalisation de ce meurtre. Le fait divers est un simulacre énonciatif. On prétend que quelqu'un parle et rapporte un événement qui est bel et bien advenu. Mais en sommes-nous si sûrs? La rumeur qui dit rendre compte de l'événementiel n'est-elle pas le modèle obscène d'une oralité dévastatrice qui entoure l'inscription de ce discours. On suppose - c'est d'ailleurs un leurre - que le fait divers, à titre de discours rapporté, structure un protocole énonciatif qui situe un sujet ayant été témoin de la scène décrite. Or, la rumeur ne parle pas d'un lieu précis. Comme la mort, elle rôde et l'enfant criminel que nous retrouvons dans ce fait divers est l'emblème d'un scéna-

2 Jean Genet, L'enfant criminel, Euvres complètes, tome 5, Paris, Gallimard, 1979, p. 387. 
rio sans auteur. L'oralité dévastatrice, c'est d'abord l'émergence d'une *voix " publique qui croit incarner la rumeur. Mais nous ignorons tout de ce meurtre à l'exception du fait divers qui prétend fonder l'événementiel. Il y a bien, à l'origine de ce fait divers, "quelqu'un" (voisin, témoin, journaliste...) qui a "vu" ce meurtre ou qui en a entendu le récit. On sait que ce fait a été traduit sous la forme d'une information dont la evoix" s'est ajoutée à la clameur médiatique. Y a-t-il "quelqu'un * cependant qui a cherché à donner à ce fait divers sa résonance sociale sans se contenter de transmettre une information anonyme? Il semble que non. Peut-être est-ce que le scripteur, qui se cache derrière ce fait divers, n'existe pas, tué par la stricte banalité de l'événement qu'il relate, assassiné par l'effroyable répétition d'un discours qui fait de la mort un enjeu marchand.

L'oralité dévastatrice à laquelle je fais référence, on peut aussi l'envisager dans une perspective qui recourt de façon plus spécifique à la psychanalyse. Nous sommes quotidiennement alimentés de discours sédatifs qui provoquent l'endormissement, qui altèrent peu à peu la faculté de juger. Le fait divers propose la consommation cannibalique de l'identique: une autophagie qui reprend constamment les mêmes arguments, qui refuse le discours au profit de la référentialité la plus plate. Être gavé, tel est le lot du lecteur qui, parcourant ce fait divers, ou y revenant obsessionnellement, cherche à combler - sans même d'ailleurs comprendre la pertinence de cette interrogation - un *vide * qui lui apparaît intolérable. On peut imaginer que l'oralité définit à cette occasion une modalité d'investissement psychique qui assemble les faits, les accumule pour mieux ainsi dénier la perception d'un vide angoissant. Pour le boulimique qui dévore le fait divers, il n'y a pas d'altérité. L'oralité dévastatrice met en scène une passion qui consiste à lire les détails de la mort de son semblable sans être cependant touché. C'est à peine si ce lecteur peut imaginer qu'un autre, là-bas, c'est-à-dire ailleurs, a tué père, mère, frères et sœurs. L'expulsion de l'abject est représentée par ce simulacre d'altérité dont le visage prend la forme de l'enfant criminel. Lire, scruter avec délectation les détails, pourtant anonymes à l'excès, du fait divers permet de banaliser la pensée. Ce n'est pas le voyeurisme qui domine dans ce fait divers puisqu'il contribuerait à faire de chacun de nous les spectateurs indiscrets d'un drame actuel. C'est surtout l'absence de vision, la perte du regard qui signe la banalité d'être témoin d'un événement qui est $\therefore$ ia fois proche et lointain. 
Étrangement, ce *fait divers - qui introduit le meurtre collectif au coeur de la réalité et dont le discours social nous assure la transmission, ce fait divers me laisse indifférent. Qu'on me comprenne bien. Le meurtre est insoutenable, l'événement est atroce. Mais je m'étonne de l'absence d'affect qui devrait accompagner tout constat objectal de deuil ou de disparition. En somme, le fait divers ne me convainc pas de la réalité de la perte d'autrui. Je ne peux pleurer un fait divers. Mais je suis prostré de douleur si je perds un être cher. Paul Virilio rappelle dans Esthétique de la disparition:

Un deuil, une impression de malheur profond peuvent, selon Bachelard, nous donner la sensation de l'instant. Ils peuvent, en tous cas, favoriser l'absence. Nous sommes affligés et nous voilà visités par quelque sensation tenace, affectant indifféremment l'un de nos organes de perception. [...] L'étrange phénomène dure parfois de longues minutes avant que tout semble à nouveau ordinaire. ${ }^{3}$

Le propos de Virilio consiste, suivant le parcours de la réflexion bachelardienne, à lier indissolublement deuil et présence. C'est en effet la perception de la perte d'autrui qui suggère sa présence. Il y a dans cette remarque de Virilio un présupposé qui fonde l'idée que la perte est singulière, que sa nomination est indicible, que la mort elle-même ne peut faire l'objet d'aucune réparation.

Si la perte, ainsi que l'entrevoit Paul Virilio, génère une accélération des sensations, une production démesurée de stimulus qui permettent de saisir la forme de l'absence, il en est tout autrement dans le fait divers analysé. L'instant présent que peut exprimer pour le survivant le deuil d'autrui n'est plus valorisé. La contemporanéité événementielle qui amène à pleurer la perte de l'être cher est délaissée au profit d'un témoignage impersonnel. Le regard ne bute pas sur l'absence, mais rencontre l'anonymat le plus radical. Cet enfant meurtrier, objet du fait divers, n'existe déjà plus. Il est l'ombre de lui-même. Il n'a de statut que dans le cadre d'un discours qui le définit comme simulacre d'une ruse marchande dont l'enjeu est la disparition même de l'affect. On ne fait plus le deuil d'autrui. On ne survit plus difficilement à l'absence. Le fait divers, présenté sous la forme d'un meurtre emblématique, autorise le sentiment d'inertie qui accompagne la lecture

3 Paul Virilio, Estbétique de la disparition, Paris, Galilée, 1989, p. 42-43. 
et la contemplation détachée d'un sacrifice. Pourquoi s'étonner de ce meurtre? "L'horreur est quotidienne ", nous chuchote la rumeur anonyme. Le fait divers, c'est le meurtre du sens.

C'est vrai, cet adolescent - mais on l'imagine encore avec les traits d'un enfant - a tué. C'est vrai, aussi, que la violence, la folie ne sont pas l'apanage des adultes et qu'il serait naif de restreindre la démence à l'âge d'homme. On peut tuer à l'adolescence sans aucune justification - même délirante - qui offre l'esquisse d'une argumentation. Comment expliquer le malaise qui me laisse traiter d'un tel sujet? Pour quelles raisons écrire sur un meurtre dont on ne sait rien? Peut-être est ce justement l'impersonnalité de mon regard qui m'amène à vouloir comprendre ce qu'est l'enfant criminel? D'autres questions, plus personnelles, surgissent et s'imposent: pourquoi les a-t-il tués? Au nom de quel drame intérieur, de quelle rage brusquement évacuée?

Le discours social, lorsqu'il prétend traiter de s'intériorité" psychique (sous le mode du désir ou de la frustration, de l'amour ou de la haine), privilégie la superficialité. L'accumulation des indices qui constituent une "preuve* anecdotique, voilà ce qui permet d'afficher l'insigne d'une vérité. On dira de cet enfant qu'il a tué son père parce qu'il ne voulait pas que ce dernier écoute une cassette qu'il avait achetée... Que sais-je encore? Or, ce fait divers relate le passage à l'acte d'un enfant devenu criminel. On ne sait rien de ce dernier. La preuve est soumise d'ores et déjà à l'effacement. La photographie nous montre un visage qui ne signifie rien, qui veut indiquer - c'est sans doute l'enjeu argumentatif préalable qui structure ce fait divers - une "histoire" sans résonance particulière. Quoique le lecteur - le voyeur scrutant ce fait divers y inscrira l'enjeu cathartique qui détermine une mise à l'écart énonciative: "ce n'est pas moi qui les ai tués" pensera-t-il, "c'est lui, cet adolescent " qui met en scène les affects qui suscitent la détestation d'autrui.

Freud énonce dans * Caractères psychopathiques sur la scène ${ }^{4}$ que la libération cathartique d'une surcharge pulsionnelle permet à l'inconscient de métaboliser des affects destructeurs et de les transformer en matériaux psychiques. En somme, Freud

4 Sigmund Freud, Standard Edition of the Complete Psycbological Works of Sigmund Freud, tome VII, sous la direction de James Strachey, Londres, Hogarth Press, [s.d.], p. 304-310. 
propose que l'inconscient, malgré la violence qu'il peut contenir, est susceptible d'être transformé en substrat représentatif. En témoignent le rêve, la scène primitive, le scénario écran: abrégés de récits qui ont pour ambition de réaliser l'effraction qui accompagne la levée du refoulement. Tout autre est la perspective dans le fait divers analysé. La transgression est ici impérative, le refoulement (ruse civilisationnelle?) rejeté au profit du passage à l'acte. Le fait divers situe donc un discours qui fait appel à une intemporelle banalité. Cet enfant est déjà condamné avant d'avoir conçu son crime. L'enfant criminel s'insère, par la trame du discours qui narre son destin, dans le cadre d'un étrange scénario où la sentence est prononcée: le jugement des adultes, tel est le prix du parricide, du matricide, du fratricide. Nous voilà au cœur de l'actualité œdipienne. Le fait divers présente une famille qui est sacrifiée par un adolescent fou. Si le meurtre peut être circonscrit par les indices chronologiques, locatifs, causatifs qui en relatent la réalisation, il n'en reste pas moins que c'est l'événementiel seul qui tient lieu de preuve aurgumentative. Il est étonnant de constater de quelle manière le fait divers fait appel à une temporalité retenue. Le crime a eu lieu. Les conséquences de ce meurtre sont longuement énumérées: "Le procureur a fait savoir qu'il ferait en sorte que le garçon soit jugé comme un adulte et non pas comme un mineur. " Nous savons que cet adolescent sera jugé. Nous ignorons pourquoi cet adolescent, pour reprendre la terrifiante expression de Genet, est devenu un *enfant criminel *.

Le psychanalyste ne peut se contenter de rumeurs ou du discours rapporté lors de l'écoute de ses analysants. C'est bien cet individu et pas un autre qui demande d'entreprendre une analyse... Mais les diverses modalités de discours rapporté fondent aussi l'essentiel du travail analytique. Je m'explique: il ne s'agit pas de savoir si le patient dit ou non la vérité, si la dimension factuelle des événements racontés est adéquate, si la chronologie est respectée. Nos souvenirs d'enfance ne font-ils pas appel à un savant processus de reconstruction mémorielle? Mais le psychanalyste est avant tout intéressé par la parole d'autrui, telle qu'elle peut être dite en cours de séance. Hors de ce cadre, la psychanalyse - comme pratique - n'existe plus. Ce n'est pas intégrisme que de l'affirmer. Il n'y a de discours qui tienne, et qui fasse intervenir la psychanalyse, qu'à la condition d'une interlocution qui suscite le désir de l'Autre. Peut-il y avoir cependant une réflexion psychanalytique qui s'intéresse au fait divers? Je répondrais par l'affirmative dans la mesure où le fait divers permet de saisir selon 
quelles modalités le discours social tolère difficilement l'intrusion de l'inconscient. À titre de discours rapporté, le fait divers propose une rationalisation factice des événements sociaux. Il met en scène une individualité souveraine - selon le modèle de l'enfant criminel - qui fait de ladolescent meurtrier un sujet responsable sans que l'on interroge plus avant les motifs de sa culpabilité:

Le seul moyen qu'auront les grandes personnes, les honnêtes gens, de sauvegarder quelque beauté morale, c'est de refuser toute pitié à des gosses qui n'en veulent pas. Car ne croyez pas, Messieurs, Mesdames, Mesdemoiselles, qu'il vous suffise de vous pencher avec sollicitude, indulgence, avec un intérêt compréhensif vers l'enfant criminel pour avoir droit a son affection et à sa gratitude: il fallait être cet enfant, il fallait, vous aussi, être le crime, et le sanctifier par une vie magnifique, c'est-à-dire par l'audace de rompre avec la toute-puissance du monde. 5

Au contraire de cette grandiosité revendiquée par Jean Genet, la banalité fonde la pseudo-logique du fait divers et l'acceptation ordinaire de la monstruosité. Tout, dans le récit de ce meurtre, est construit narrativement autour de la présence des témoins qui valident la concrétion de ce passage à l'acte. Le meurtre a eu lieu. Il faut constater l'horreur et se contenter d'être les lecteurssurvivants d'un assassinat qu'il faudra oublier.

Il arrive, c'est vrai, que des enfants tuent, aimantés par la démence, leurs parents, leurs frères et sœurs. Le nier serait tout simplement idiot. Mais il arrive aussi que des enfants tuent afin de justifier, par identification projective, leur monstruosité, c'est-àdire la difformité qui, aux yeux du monde des adultes, caractérise l'enfant criminel. C'est l'argument que développe Genet dans un écrit dont la luminosité est impitoyable. On peut constater, dans cet éloge de l'enfant criminel que formule Genet, quelque chose d'assez troublant: l'acceptation d'une Loi d'autant plus dure et impersonnelle qu'elle désire le châtiment.

Le fait divers élabore une esthétique de la disparition. Les événements rapportés sont anecdotiques et font référence à un acte dont tout pathos semble évacué. Faut-il voir dans cette absence d'émotion un symptôme civilisationnel: la crainte d'être ému et touché par l'Autre? Une des caractéristiques de ce fait divers consiste à instaurer le retrait affectif comme mode de 
pensée. Cet enfant commet un meurtre. La communauté sociale y voit la simple expression d'un raptus. La pulsion homicide n'est pas évoquée sous la forme d'un projet élaboré de longue date. Le fait divers présente un meurtre fondé sur l'absence d'attachement.

Un adolescent tue sa famille. Par cette action radicale, il abolit toute filiation. La singularité folle de ce geste destructeur désigne un sujet dont la monstruosité est révélée. Il est question dans ce fait divers de l'enfance: expression dont je retiens la fluidité puisqu'elle détermine la frontière qui sépare arbitrairement du monde des adultes. Lorsque Jean Genet évoque la figure de l'enfant criminel, il y inscrit la dure Loi du châtiment qui est associée à l'enfance:

Or, si des enfants ont l'audace de vous dire non, châtiez-les. Soyez durs afin qu'ils ne vous ménagent pas. Mais depuis longtemps, vous trichez. À vos Tribunaux, à vos Cours, vous n'observez plus déjà le cérémonial du rituel - non que vous l'ayez remplacé par une cruauté plus intime, une cruauté en veston, si j'ose dire - mais, par un grave laisser-aller vous venez au prétoire avec une robe rapiécée dont les revers parfois ne sont même plus de soie, mais de rayonne ou de lustrine. [...] Il ll'enfant criminell est sur le point de vous offrir une peu de majesté qu'il sait obtenir d'une séance plus solennelle où il comparaît en secret cependant que vous continuez sous ses yeux votre enfantin simulacre. ${ }^{6}$

L'enfant criminel respecte la loi. Cet écrit de Genet fait jouer les motifs de la faute et de la sanction sacrificielle. Ce n'est pas, indique encore Genet, qu'il soit juste ou choquant de condamner. L'extrême démesure de la pensée de Genet propose une accentuation paroxystique du châtiment qui justifie l'anticipation de la Loi. Ainsi Genet écrira:

Mais le jeune criminel refuse déjà l'indulgente compréhension, et sa sollicitude, d'une société contre qui il vient de s'insurger en commettant son premier délit. Ayant à 15 ou 16 ans, ou plus tôt, acquis une majorité que les braves gens n'auront pas encore à 60 , il méprise leur bonté. Il exige que sa punition soit sans douceur. Il exige d'abord que les termes qui la définissent soient le signe d'une cruauté majeure. C'est avec une sorte de honte que l'enfant avoue qu'on vient de l'acquitter ou qu'on le condamne à une peine légère. Il souhaite la rigueur. Il l'exige. ${ }^{7}$

$6 \quad$ Ibid., p. 391-392.

7 Ibid., p. 382. 
96

Le caractère implacable de la Loi est si violent qu'il oblige instantanément l'enfant à se muer en adulte persécuteur. Telle est la signification implicite de ce texte de Genet. Mais on peut aussi se demander, dans la perspective qu'adopte Genet, quel Idéal secret justifie l'acceptation du châtiment. Deviendra criminel cet enfant dont le crime sera exemplifié et qui aura droit de cité pour être exclu de la communauté. Deviendra criminel ce personnage mi-adulte mi-enfant qui cherche la condamnation et refuse l'acquittement. Que la Loi soit dure et vaine, que le sacrifice soit inexpiable et qu'il suscite la haine éperdue des adultes, voilà la destinée que Jean Genet trace à cet enfant criminel. On ne sera donc pas surpris de lire sous sa plume:

En lui-même il entretient le rêve que la forme qu'elle prendra [la punition] sera un enfer terrible, et la maison de correction l'endroit du monde d'où l'on ne revient pas. En effet, on n'en revenait pas. En en sortant on était autre. ${ }^{8}$

Le fait divers ne connaît pas cette violente altérité. Pour que cette dernière soit dicible - disons, pour qu'elle ait une résonance qui lui octroie une signification culturelle - , il faudrait que l'acte criminel instaure une transgression. Or, ici, la banalité règne. Si Genet, dans le cadre d'un discours lyrique, situe l'Idéal qui sous-tend sa perception de l'enfant criminel, il faut constater que le fait divers, lui, ne laisse parler que l'absence. Singulière cette absence qui nous transforme en témoins évanescents d'un meurtre dont les motifs sont oubliés.

Ibid. 